Mon. Not. R. Astron. Soc. 000,111(2007) Printed 30 October $2018 \quad$ (MN LATEX style file v2.2)

\title{
Desorption From Interstellar Ices
}

\author{
J. F. Roberts ${ }^{\star}$, J. M. C. Rawlings, S. Viti and D. A. Williams \\ Department of Physics \& Astronomy, University College London, Gower Street, London WC1E 6BT
}

30 October 2018

\begin{abstract}
The desorption of molecular species from ice mantles back into the gas phase in molecular clouds results from a variety of very poorly understood processes. We have investigated three mechanisms; desorption resulting from $\mathrm{H}_{2}$ formation on grains, direct cosmic ray heating and cosmic ray induced photodesorption. Whilst qualitative differences exist between these processes (essentially deriving from the assumptions concerning the species-selectivity of the desorption and the assumed threshold adsorption energies, $E_{\mathrm{t}}$ ) all three processes are found to be potentially very significant in dark cloud conditions. It is therefore important that all three mechanisms should be considered in studies of molecular clouds in which freeze-out and desorption are believed to be important.

Employing a chemical model of a typical static molecular core and using likely estimates for the quantum yields of the three processes we find that desorption by $\mathrm{H}_{2}$ formation probably dominates over the other two mechanisms. However, the physics of the desorption processes and the nature of the dust grains and ice mantles are very poorly constrained. We therefore conclude that the best approach is to set empirical constraints on the desorption, based on observed molecular depletions - rather than try to establish the desorption efficiencies from purely theoretical considerations. Applying this method to one such object (L1689B) yields upper limits to the desorption efficiencies that are consistent with our understanding of these mechanisms.
\end{abstract}

Key words: astrochemistry - molecular processes - stars:formation - ISM: abundances - ISM: dust - ISM: molecules.

\section{INTRODUCTION}

In cold dark interstellar clouds, heavy molecules accumulate onto dust grains, forming icy mantles on their surfaces. This process, known as freeze-out, occurs on a timescale of a few times $10^{9} n_{\mathrm{H}}^{-1}$ years (where $n_{\mathrm{H}}=n(\mathrm{H})+2 n\left(\mathrm{H}_{2}\right)$ is the total hydrogen nucleon number density) in the absence of desorption. This is much less than the expected lifetime of a typical molecular cloud so, if freeze-out was unlimited, we would expect the majority of observations to show no evidence for heavy gas phase species. However, observations of molecules such as $\mathrm{CO}$ in dark clouds, for example L1689B, TMC1-CP and L134N (Lee at al. 2003; Smith et al. 2004; Wakelam et al. 2006), indicate that desorption processes must be operating for mantle growth to be limited.

Although this conclusion has been accepted for over 20 years, it is still not fully understood how this desorption occurs. Many possible mechanisms have been proposed, most of which require impulsive heating of grains

* E-mail: jfr@star.ucl.ac.uk which can be caused by (a) direct impact of cosmic rays (Hasegawa \& Herbst 1993; Hartquist \& Williams 1990; Léger et al. 1985), (b) X-rays (Léger et al. 1985), (c) ultraviolet photons induced by cosmic rays (cosmic ray photodesorption) (Hartquist \& Williams 1990; Dulev et al. 1989), or (d) exothermic reactions occurring on the grain surface (Allen \& Robinson 1975; Garrod et al. 2007), in particular the formation of molecular hydrogen (Willacy et al. 1994a; Dulev \& Williams 1993). Molecules can either be desorbed by classical evaporation (Léger et al. 1985) or by chemical explosions (Shen et al. 2004; Shalabiea \& Greenberg 1994; Léger et al. 1985). These chemical explosions can only occur if the mantle has previously been irradiated with ultra-violet radiation which creates radicals. If the grain temperature is raised to $\sim 27 \mathrm{~K}$, the radicals become mobile and then undergo explosive reactions capable of expelling the entire grain mantle. We will not consider chemical explosions in this paper because in dark clouds with $A_{V}>5$ magnitudes it is unlikely that the grain mantles will have received sufficient UV irradiation (Léger et al. 1985), and any radicals that do form are likely to be hydrogenated due to the high abundance of hydrogen atoms present (Willacy \& Millar 1998). 
Recent chemical models of dark clouds tend to include only desorption via thermal evaporation (which is negligible for dark clouds with temperatures of $10 \mathrm{~K}$ ) and/or direct heating by cosmic rays, using the formulation given by Hasegawa \& Herbst (1993) (hereafter HH93) (Ruffle \& Herbst 2000; Roberts et al. 2004). This paper, therefore, aims to test the assumption that desorption via direct cosmic ray heating is the only effective non-thermal desorption mechanism relevant to dark molecular cores. In this study we adopt the model of a molecular cloud as being composed of an ensemble of dense (dark) cores in a more diffuse background (Garrod et al. 2006). Ices are probably only present in the cores. We set out to test the desorption efficiencies, by including in an existing model of dark cloud chemistry three desorption mechanisms (desorption resulting from $\mathrm{H}_{2}$ formation on grains, direct cosmic ray heating and cosmic ray photodesorption) that have been formulated in the literature, to find their relative importance in dark cores. We believe these three mechanisms are likely to be the most important in this situation, in the absence of any nearby X-ray sources in the molecular cloud.

We note that there already exist several other studies which model these three desorption mechanisms, for example Willacy et al. (1994b) (hereafter WRW94) performed a study very close to our own. However, our work differs to that of WRW94 because we try to take a simpler approach; by modelling a static cloud rather than a collapsing one we can easily see the effects of adding desorption to our chemical model. We also look further into the assumptions made about the ability of each desorption mechanism to desorb molecules with higher adsorption energies, such as $\mathrm{H}_{2} \mathrm{O}$ and $\mathrm{NH}_{3}$ (Section 4.3). Most importantly, we attempt to constrain the efficiency of each mechanism by comparing our results to observations of $\mathrm{CO}$ depletion in star-forming regions.

The paper is structured as follows: In Section 2 we give a brief summary of these desorption mechanisms and in Section 3 we describe the model. The results are given in Section 4. and are discussed by comparing them to existing models in Section 5 and observations in Section 6] Concluding remarks are given in Section 7.

\section{DESORPTION MECHANISMS}

\subsection{Desorption resulting from $\mathrm{H}_{2}$ formation}

It has long been suggested that the energy released from exothermic reactions on grain surfaces can release energy capable of desorbing mantle species (Allen \& Robinson 1975). In particular, laboratory experiments on graphite substrates suggest that a non-negligible fraction, perhaps up to $40 \%$ (Creighan, Perrv \& Price 2006), (F. Islam, private communication) of the $\sim 4.5 \mathrm{eV}$ released in the surface formation of molecular hydrogen is transfered to the grain surface. This leads to local heating which may thermally desorb weakly bound mantle species (Duley \& Williams 1993), although the extent to which the temperature rises transiently is not well determined, and depends on the local conductivity. In amorphous materials, the temperature rise may be relatively large.

Such a process may be selective, depending on the tem- perature achieved as a result of the energy deposition. In previous work (Dulev \& Williams 1993; Willacy et al. 1994a b), the conservative assumption was made that only the most volatile species (such as $\mathrm{CO}, \mathrm{N}_{2}, \mathrm{NO}, \mathrm{O}_{2}, \mathrm{C}_{2}$ and $\mathrm{CH}_{4}$ ) would be desorbed during the transient heating, corresponding to a threshold adsorption energy of $E_{\mathrm{t}}=1210 \mathrm{~K}$. Initially, we shall adopt the same threshold and range of volatile species, but we shall also consider the effects of variations from that value in Section 4.3

The rate of desorption by this process for species $i$ is given by:

$$
R_{\mathrm{hf}}=\varepsilon R_{\mathrm{H}_{2}} M_{\mathrm{s}}(i, t) \quad \mathrm{cm}^{-3} \mathrm{~s}^{-1}
$$

where $R_{\mathrm{H}_{2}}$ is the rate of $\mathrm{H}_{2}$ formation on grains. $R_{\mathrm{H}_{2}}$ is proportional to the available grain surface area and the sticking/reaction probability to form $\mathrm{H}_{2}$ and is empirically constrained (at $\mathrm{T}=10 \mathrm{~K}$ ) to be given by:

$$
R_{\mathrm{H}_{2}}=3.16 \times 10^{-17} n(\mathrm{H}) n_{\mathrm{H}} \quad \mathrm{cm}^{-3} \mathrm{~s}^{-1}
$$

where $n(\mathrm{H})$ is the number density of atomic hydrogen and $n_{\mathrm{H}}$ is the total hydrogen nucleon density. $M_{\mathrm{s}}(i, t)$ is the fraction of the mantle consisting of species $i$, calculated selfconsistently as a function of time. $\varepsilon$ is an efficiency parameter such that $\varepsilon M_{\mathrm{s}}(i, t)$ gives the number of molecules of species $i$ desorbing per $\mathrm{H}_{2}$ molecule formed. The efficiency of this process is uncertain, but we can make a rough upper estimate as follows: If we assume that $40 \%(\sim 2 \mathrm{eV})$ of the energy released during each $\mathrm{H}_{2}$ formation is transfered to the grain, and given that the adsorption energies of the species we consider are $\sim 1000 \mathrm{~K}\left(\sim 8.2 \times 10^{-2} \mathrm{eV}\right)$, we would expect that a maximum of $\sim 20$ molecules could be desorbed every time an $\mathrm{H}_{2}$ molecule is formed. It is likely, however, that this process is less efficient, because such a high value for $\varepsilon$ would prevent the build-up of any mantle material until very late times (Willacy \& Millar 1998). We therefore run our model with values of $\varepsilon$ ranging from 0.01 to 1.0 , and present results for $\varepsilon=0.01$ and 0.1 .

\subsection{Desorption by direct cosmic ray heating}

In this paper, the rate of desorption by direct cosmic ray heating is calculated by simply considering the number of molecules capable of being desorbed per cosmic ray impact. This rate is different to that adopted in other models (see the discussion in Section 5.4).

As with desorption via $\mathrm{H}_{2}$ formation, this process is believed to be selective (Willacv et al. 1994b), so only the volatile species (CO, etc.) are expected to be desorbed, at a rate given by:

$$
R_{\mathrm{cr}}=F_{\mathrm{cr}}\left\langle\pi a_{\mathrm{g}}^{2} n_{\mathrm{g}}\right\rangle \phi M_{\mathrm{s}}(i, t) \quad \mathrm{cm}^{-3} \mathrm{~s}^{-1} .
$$

where $F_{\text {cr }}$ is the flux of cosmic rays, $\phi$ is an efficiency parameter such that $\phi M_{\mathrm{s}}(i, t)$ is the number of molecules released per cosmic ray impact, $a_{\mathrm{g}}$ is the grain radius and $n_{\mathrm{g}}$ is the number density of grains. We have used a value for the total grain surface area per $\mathrm{cm}^{3},\left\langle\pi a_{\mathrm{g}}^{2} n_{\mathrm{g}}\right\rangle$, of $2.4 \times 10^{-22} n_{\mathrm{H}} \mathrm{cm}^{-1}$, consistent with Rawlings et al. (1992) if we use a value for the depletion coefficient of $\sim 1.0$.

Léger et al. (1985) showed that the iron nuclei component of cosmic rays should be the most effective in heating the grain, and therefore we adopt for the value for $F_{\text {cr }}$ of $2.06 \times 10^{-3} \mathrm{~cm}^{-2} \mathrm{~s}^{-1}$ which is an estimate of the 
Desorption From Interstellar Ices

iron fraction of the canonical cosmic ray flux. It is based on the proton cosmic ray flux in the interstellar medium given by Morfill et al. (1976), and the iron to proton ratio of $1.6 \times 10^{-4}$ (estimated by Léger et al. (1985)) .

An estimate for $\phi$ can be taken from Léger et al. (1985) who worked out the evaporation rate of $\mathrm{CO}$ per grain resulting from both whole-grain and spot-grain heating by cosmic rays. For spot-grain heating, they calculated that $6 \times 10^{4}$ $\mathrm{CO}$ molecules would be released per cosmic ray impact, independent of grain size. For whole grain heating, however, the result was found to strongly depend on grain size, dominating spot-grain heating for $a_{\mathrm{g}} \leqslant 2500 \AA$. We have tested this mechanism with values of $\phi$ ranging from $10^{2}$ to $10^{6}$, but we only present the results for $\phi=10^{5}$ to be consistent with the lower estimate predicted by spot-grain heating. Since the volatile species make up approximately $40 \%$ of the grain mantle, using $\phi=10^{5}$ implies that the number of molecules released per cosmic ray impact is $\phi M_{\mathrm{S}}(i) \approx 4 \times 10^{4}$.

\subsection{Cosmic ray induced photodesorption}

As cosmic rays travel through a molecular core, they ionise and excite the absorbing gas. Prasad \& Tarafdar (1983) investigated the excitation of the Lyman and Werner systems of the hydrogen molecule, caused by collisions with either primary cosmic ray particles or secondary electrons released by cosmic ray ionisation. They found that emissions resulting from these excitations may be capable of maintaining a chemically significant level of UV photon flux in the interiors of dark clouds, where the interstellar UV radiation is heavily extinguished $\left(A_{V} \geqslant 5\right)$.

When a UV photon impinges upon the mantle of a grain, it is most likely to be absorbed by $\mathrm{H}_{2} \mathrm{O}$ which may then be dissociated into $\mathrm{H}$ and $\mathrm{OH}$ (Hartquist \& Williams 1990). The energy of the dissociation products cause local heating, capable of desorbing nearby molecules. This process is non-selective, and proceeds at a rate given by:

$$
R_{\text {crpd }}=F_{\mathrm{P}}\left\langle\pi a_{\mathrm{g}}^{2} n_{\mathrm{g}}\right\rangle Y M_{\mathrm{s}}(i, t) \quad \mathrm{cm}^{-3} \mathrm{~s}^{-1} .
$$

where $F_{\mathrm{P}}$ is the photon flux and $Y$ is the yield per photon. Prasad \& Tarafdar (1983) estimated $F_{\mathrm{P}}$ to be just $1350 \mathrm{~cm}^{-2} \mathrm{~s}^{-1}$, but here we use a value of $4875 \mathrm{~cm}^{-2} \mathrm{~s}^{-1}$ taken from equation (21) of Cecchi-Pestellini \& Aiello (1992) 1. This value is much higher than the estimate of Prasad \& Tarafdar (1983) because it explicitly includes the contribution to the excitation rate of $\mathrm{H}_{2}$ by cosmic ray protons, not just the secondary electrons. We present the results for $Y=0.1$, as estimated by Hartquist \& Williams (1990), but as this value is uncertain we also ran the model with values of $Y$ ranging from $1 \times 10^{-3}$ to 100 .

Obviously, the relative importance of these mechanisms depends upon the values used for $\varepsilon, \phi$ and $Y$, which can be treated as free parameters. In this paper, we present the results for the values stated above, and in Section 6 we derive upper limits for these parameters based on observations.

1 This is consistent with using a cosmic ray ionisation rate, $\zeta$, of $1.3 \times 10^{-17} \mathrm{~s}^{-1}$.

\section{THE MODEL}

In order to see clearly the relative importance of the various desorption mechanisms for the interstellar chemistry of molecular cores, we have used a simple one-point model of a static, isothermal dark cloud, at a density of $n_{\mathrm{H}}=10^{5} \mathrm{~cm}^{-3}$ and a temperature of $10 \mathrm{~K}$. The core has a visual extinction, $A_{V}$, of 10 magnitudes, and a radius of $\sim 0.05 \mathrm{pc}$, implying a mass of $1 \mathrm{M}_{\odot}$.

\subsection{The chemical model}

The model, adapted from Viti et al. (2004), includes gasphase reactions, freeze-out, surface reactions and desorption, with 127 gas phase and 40 mantle species. The gas phase reaction network consists of 1741 reactions from the UMIST Rate99 database (Le Teuff et al. 2000). Photoreactions are included, which take into account both the external interstellar radiation field and the internal cosmic ray induced UV field. Both direct and indirect ionisation by cosmic rays are also included, using a cosmic ray ionisation rate, $\zeta$, of $1.3 \times 10^{-17} \mathrm{~s}^{-1}$. The hydrogen atom abundance (that drives the $\mathrm{H}_{2}$ formation desorption mechanism) is determined selfconsistently in the chemical network. The species and reactions are taken from the model as in Viti et al. (2004), which describes the rich chemistry of hot cores. The number of species and elements should therefore be more than sufficient to describe the low mass case in this paper. Freezeout and grain chemistry are described in Section 3.2

The initial conditions are atomic, apart from carbon which is all singly ionised, and half the hydrogen nuclei are in the form of $\mathrm{H}_{2}$. The initial elemental abundances are taken from Sofia \& Mever (2001).

\section{$3.2 \quad$ Freeze-out and grain chemistry}

The rate of accretion onto grains of species $i\left(\right.$ in $\mathrm{cm}^{-3} \mathrm{~s}^{-1}$ ) is given by (Rawlings et al. 1992):

$\frac{d n(i)}{d t}=-4.57 \times 10^{4}\left\langle\pi a_{\mathrm{g}}^{2} n_{\mathrm{g}}\right\rangle T^{1 / 2} C S(i) m(i)^{-1 / 2} n(i)$

where $T$ is the gas temperature, $m(i)$ is the mass of species $i$ in atomic mass units, $n(i)$ is the number density of species $i,\left\langle\pi a_{\mathrm{g}}^{2} n_{\mathrm{g}}\right\rangle$ has the same definition as in Section 2 and $S(i)$ is the sticking coefficient of species $(i)$. Following WRW94, we have used $S(i)=0.3$ for all species.

$C$ is a factor which takes into account electrostatic effects:

$$
\begin{array}{rlrl}
C & =1 & & \text { for neutral species } \\
& =1+\left(16.71 \times 10^{-4} / a_{\mathrm{g}} T\right) & & \text { for singly charged } \\
& & \text { positive ions }
\end{array}
$$

The only surface reactions that occur are hydrogenation of certain unsaturated species, and dissociative recombination of molecular ions. These reactions are assumed to occur instantly and the products remain on the grain surface until they are desorbed by one of the processes named above. $\mathrm{H}_{2}$ is an exception in that it is assumed to desorb immediately because of the high exothermicity of the reaction, as is indicated by experimental studies (Creighan, Perry \& Price 2006). 


\section{RESULTS}

By comparing the rate coefficients given for each of the desorption mechanisms, if we use $\varepsilon=0.01, \phi=10^{5}$ and $Y=0.1$ at a density of $n_{\mathrm{H}}=10^{5} \mathrm{~cm}^{-3}$, desorption via $\mathrm{H}_{2}$ formation will dominate if the equilibrium atomic hydrogen density is greater than $\sim 0.4 \mathrm{~cm}^{-3}$. If $\varepsilon$ is as high as 0.1 then this mechanism will always be dominant.

Figures 1 and 2 compare the effect of the different desorption mechanisms in a static cloud at a density of $10^{5} \mathrm{~cm}^{-3}$ for selected observable molecules. In general, desorption has the most significant effect on gas phase abundances after approximately $10^{6}$ years, which is when the molecules begin to freeze-out. Each of the desorption mechanisms are effective enough to be able to compete with freeze-out at late times, preventing full depletion of species onto grains. The exceptions are the sulphur bearing species, $\mathrm{H}_{2} \mathrm{~S}$ and CS, whose freeze-out is only prevented by the nonselective cosmic ray photodesorption. This mechanism is also able to enhance abundances of molecules such as $\mathrm{NH}_{3}$ and $\mathrm{H}_{2} \mathrm{~S}$ at times as early as $10^{5}$ years. For the case of $\mathrm{NH}_{3}$ this is because the $\mathrm{NH}_{3}$ in the mantle builds up relatively quickly (compared to molecules like CO), so direct desorption by this mechanism can proceed close to its maximum rate. However, since the $\mathrm{H}_{2}$ formation and direct cosmic ray heating mechanisms are not able to desorb $\mathrm{NH}_{3}$ directly, desorption via these mechanisms can only affect $\mathrm{NH}_{3}$ once enough NO has been desorbed to enhance the gas phase production (via the reaction $\mathrm{NO}+\mathrm{NH}_{3}^{+} \rightarrow \mathrm{NH}_{3}+\mathrm{NO}^{+}$).

For most of the species shown in Figures 1 and 2 desorption via $\mathrm{H}_{2}$ formation with $\varepsilon=0.1$ is the most effective mechanism, being able to produce equilibrium abundances up to an order of magnitude greater than the other mechanisms. However, there are several molecules $\left(\mathrm{NH}_{3}, \mathrm{H}_{2} \mathrm{~S}\right.$ and $\mathrm{CS}$ ), for which cosmic ray induced photodesorption is more effective, even though desorption via this mechanism proceeds at a rate more than ten times slower than $\mathrm{H}_{2}$ formation with $\varepsilon=0.1$. This is because we have assumed desorption via $\mathrm{H}_{2}$ formation is selective, only being able to desorb molecules with adsorption energies less than $1210 \mathrm{~K}$ (CO, $\mathrm{NO}, \mathrm{N}_{2}, \mathrm{O}_{2}, \mathrm{C}_{2}$ and $\mathrm{CH}_{4}$ ). Evidently, the higher gas phase abundances of these molecules caused by selective desorption do not have a significant effect on the gas phase chemistry of molecules like $\mathrm{H}_{2} \mathrm{~S}$ and $\mathrm{CS}$, so their freeze-out is not prevented by selective mechanisms.

In the following subsections we look at the effectiveness of each mechanism under different conditions, by varying the density and the initial atomic hydrogen density. We also discuss further the effects of selectivity in Section 4.3

\subsection{Varying the density}

When the model was run at a density of $n_{\mathrm{H}}=10^{6} \mathrm{~cm}^{-3}$, it was found that the equilibrium abundances of the desorbed species were reduced approximately by a factor of 10 compared to the equivalent runs at $10^{5} \mathrm{~cm}^{-3}$. Given that $n(\mathrm{H})$ and $M_{\mathrm{s}}$ appear to show little variation in their equilibrium values at different densities, the rates for each desorption mechanism should not depend on density. However, the rate for freeze-out has a direct linear dependence on density, so it is expected that the equilibrium abundances of desorbed species should scale roughly as $n_{\mathrm{H}}^{-1}$, which would explain the reduction in equilibrium abundances found in the results.

\subsection{Varying the initial atomic hydrogen density}

Since desorption via $\mathrm{H}_{2}$ formation depends on the abundance of atomic hydrogen, it would be expected that varying the initial ratio of atomic to molecular hydrogen could also influence how efficient this mechanism is. However, even when the model was run with all hydrogen in the form of $\mathrm{H}_{2}$ initially, after $10^{5}$ years the abundance of atomic hydrogen had reached the same value $\left(n(\mathrm{H}) \sim 0.4 \mathrm{~cm}^{-3}\right)$ as in the previous runs (which had only half the hydrogen nuclei in $\mathrm{H}_{2}$ initially), so there was no noticeable difference in the desorption via $\mathrm{H}_{2}$ formation rate.

\subsection{Varying the threshold adsorption energy for selective mechanisms}

So far we have assumed that the selective desorption mechanisms $\left(\mathrm{H}_{2}\right.$ formation and direct cosmic ray heating) are only capable of desorbing molecules with adsorption energies less than or equal to the value $E_{\mathrm{t}}=1210 \mathrm{~K}$. This number was chosen to be consistent with the study by WRW94, who assumed that only $\mathrm{CO}, \mathrm{N}_{2}, \mathrm{C}_{2}, \mathrm{O}_{2}$ and NO could be desorbed by these processes. $\mathrm{CH}_{4}$ also has an adsorption energy of less than $1210 \mathrm{~K}$, so we have also included $\mathrm{CH}_{4}$ as a 'volatile' species. Although Léger et al. (1985) calculated that mantles composed purely of refractory ices such as $\mathrm{CO}_{2}$, $\mathrm{H}_{2} \mathrm{CO}, \mathrm{HCN}, \mathrm{NH}_{3}$ and $\mathrm{H}_{2} \mathrm{O}$ are unlikely to be affected by these mechanisms, they predicted that if these molecules are mixed with volatile species then spot heating processes may be able to raise the temperature of the grains enough to desorb these refractory molecules. Since the $\mathrm{H}_{2}$ formation and direct cosmic ray heating mechanisms are both capable of spot-heating, we thought it was necessary to investigate the effect of varying $E_{\mathrm{t}}$.

Figures 3 and 4 show the effect of varying $E_{\mathrm{t}}$ for the $\mathrm{H}_{2}$ formation mechanism with $\varepsilon=0.01$. The adsorption energies for each mantle species were taken from Aikawa et al. (1997), apart from $\mathrm{H}_{2} \mathrm{O}$ which was taken from Fraser et al. (2001). Some of the molecules, such as $\mathrm{CO}, \mathrm{CH}_{4}$ and $\mathrm{H}_{2} \mathrm{CO}$ are relatively unaffected, even when $E_{\mathrm{t}}$ is so high $(10000 \mathrm{~K})$ that all molecules can be desorbed. In the case of $\mathrm{H}_{2} \mathrm{CO}$ which has an adsorption energy of $1760 \mathrm{~K}$ (Aikawa et al. 1997) this may seem rather surprising. The behaviour can be explained by the fact that, for the model and parameters that we have considered, gas-phase formation of $\mathrm{H}_{2} \mathrm{CO}$ (via $\mathrm{CH}_{3}+\mathrm{O}$ ) dominates over desorption. On the other hand, molecules such as $\mathrm{NH}_{3}$ and HCN show differences in abundances of more than one order of magnitude when they reach equilibrium. These differences can be explained as follows:

(i) $\mathrm{NH}_{3}$ : The equilibrium fractional abundance of $\mathrm{NH}_{3}$ increases from $1.4 \times 10^{-9}$ to $2.5 \times 10^{-8}$ when $E_{\mathrm{t}}$ increases from $3000 \mathrm{~K}$ to $4000 \mathrm{~K}$. This change is simply because the adsorption energy of $\mathrm{NH}_{3}$ is $3080 \mathrm{~K}$, so for $E_{\mathrm{t}} \geqslant 4000 \mathrm{~K}$, $\mathrm{NH}_{3}$ can be desorbed directly. For $E_{\mathrm{t}} \leqslant 4000 \mathrm{~K}$, the main production of $\mathrm{NH}_{3}$ is through the reaction $\mathrm{NH}_{3}^{+}+\mathrm{NO} \rightarrow$ $\mathrm{NO}^{+}+\mathrm{NH}_{3}$.

(ii) $\mathrm{HCN}$ : The equilibrium fractional abundance of $\mathrm{HCN}$ increases from $9.5 \times 10^{-11}$ to $2.0 \times 10^{-9}$ when $E_{\mathrm{t}}$ increases 

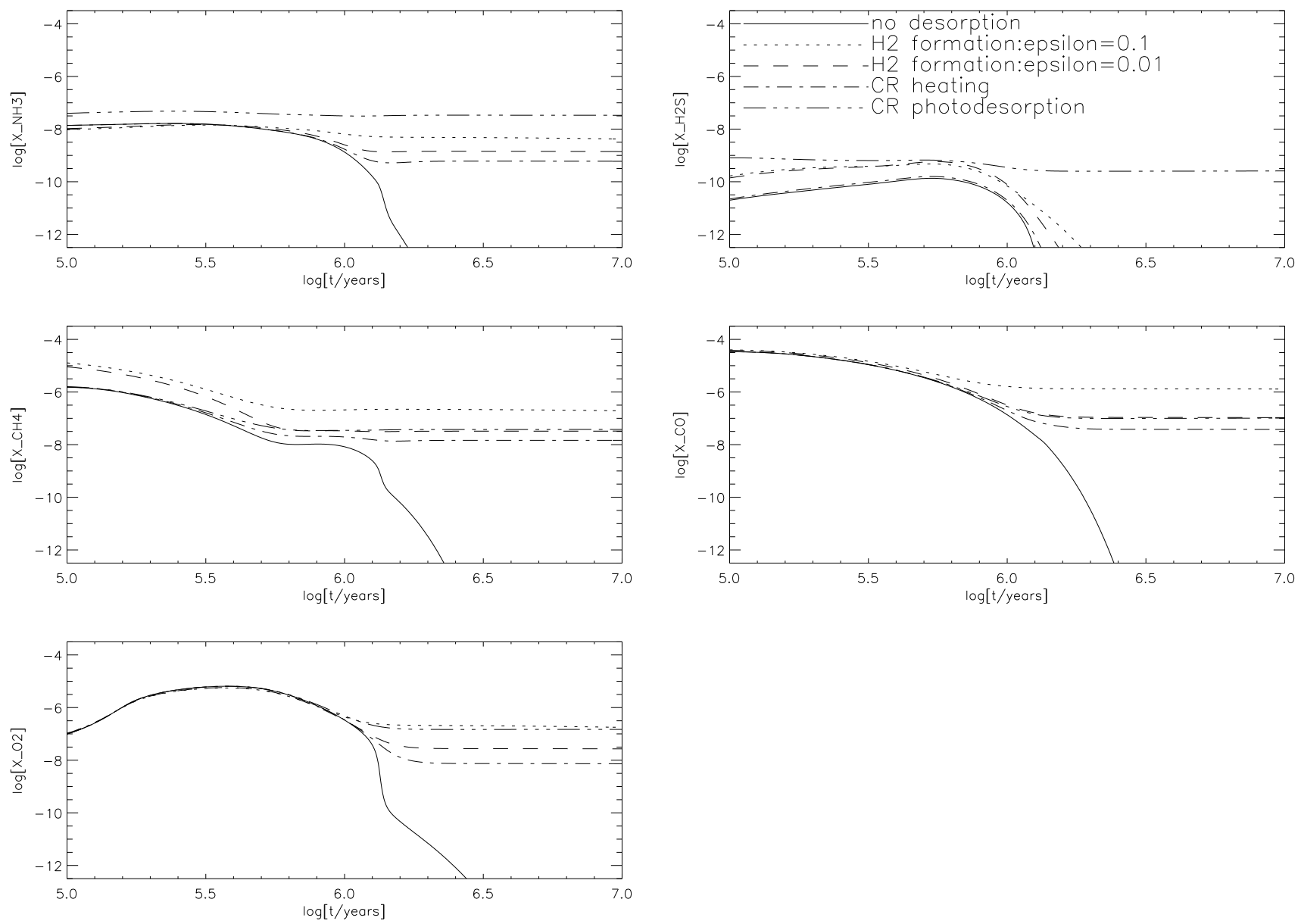

Figure 1. The time evolution of the molecules $\mathrm{NH}_{3}, \mathrm{H}_{2} \mathrm{~S}, \mathrm{CH}_{4}, \mathrm{CO}$ and $\mathrm{O}_{2}$ in a static cloud of density $n_{\mathrm{H}}=10^{5} \mathrm{~cm}^{-3}, T=10 \mathrm{~K}$, at a point with $A_{V}=10 \mathrm{mag}$. The abundances of species relative to hydrogen are shown as a function of time in years. The different curves compare the evolution of the species with no desorption, desorption via $\mathrm{H}_{2}$ formation with $\varepsilon=0.1$ and 0.01 , desorption via direct cosmic ray heating and cosmic ray photodesorption (see key).

from $3000 \mathrm{~K}$ to $4000 \mathrm{~K}$. The adsorption energy of $\mathrm{HCN}$ is actually greater than $4000 \mathrm{~K}$, so this is not a consequence of direct $\mathrm{HCN}$ desorption. In fact, it is the direct desorption of $\mathrm{NH}_{3}$ that produces the $\mathrm{HCN}$ via the reaction $\mathrm{NH}_{3}+\mathrm{CN} \rightarrow$ $\mathrm{HCN}+\mathrm{NH}_{2}$ when $E_{\mathrm{t}}$ is greater than $4000 \mathrm{~K}$. Below this energy, the main route of production of $\mathrm{HCN}$ is the reaction $\mathrm{H}+\mathrm{H}_{2} \mathrm{CN} \rightarrow \mathrm{HCN}+\mathrm{H}_{2}$

For CS and $\mathrm{H}_{2} \mathrm{~S}$, freeze-out is only prevented if $E_{\mathrm{t}}$ is greater than $2000 \mathrm{~K}$. This is because at this energy both CS and $\mathrm{H}_{2} \mathrm{~S}$ can be directly desorbed. For CS if $E_{\mathrm{t}}$ is increased further from $2000 \mathrm{~K}$ to $3000 \mathrm{~K}$, the equilibrium abundance of CS increases again by approximately an order of magnitude. This is due to the direct desorption of $\mathrm{H}_{2} \mathrm{CS}$ at $2250 \mathrm{~K}$, which can increase the CS abundance through the reaction $\mathrm{C}^{+}+\mathrm{H}_{2} \mathrm{CS} \rightarrow \mathrm{CS}+\mathrm{CH}_{2}^{+}$.

\section{COMPARISON WITH EXISTING MODELS AND FORMULATIONS}

We first of all compare our results to those of WRW94, which is the closest previous study to our own, then we consider two other studies, Willacy \& Millar (1998) and Hartquist \& Williams (1990) (hereafter WM98 and HW90 respectively), whose conclusions differed to ours. We also discuss various assumptions that are made in most chemical models of molecular clouds in which desorption is included, with particular reference to the formulation of desorption by direct cosmic ray heating by HH93.

\subsection{Comparison to WRW94}

WRW94 investigated each of the desorption mechanisms discussed above, in the case of a collapsing cloud. They concluded that the only significant mechanism was desorption arising from $\mathrm{H}_{2}$ formation on grains if it was assumed to be non-selective, using values for $\varepsilon$ of 0.1 and 0.8 . Since we have found that, for a static cloud, all desorption mechanisms are significant and can prevent complete freeze-out, it is therefore worth checking that our model agrees with that of WRW94 when it is run under similar conditions.

To simulate a collapse, as in WRW94, we have used a spherically symmetric, isothermal model, undergoing a freefall collapse. The collapse is modified by a retardation factor 

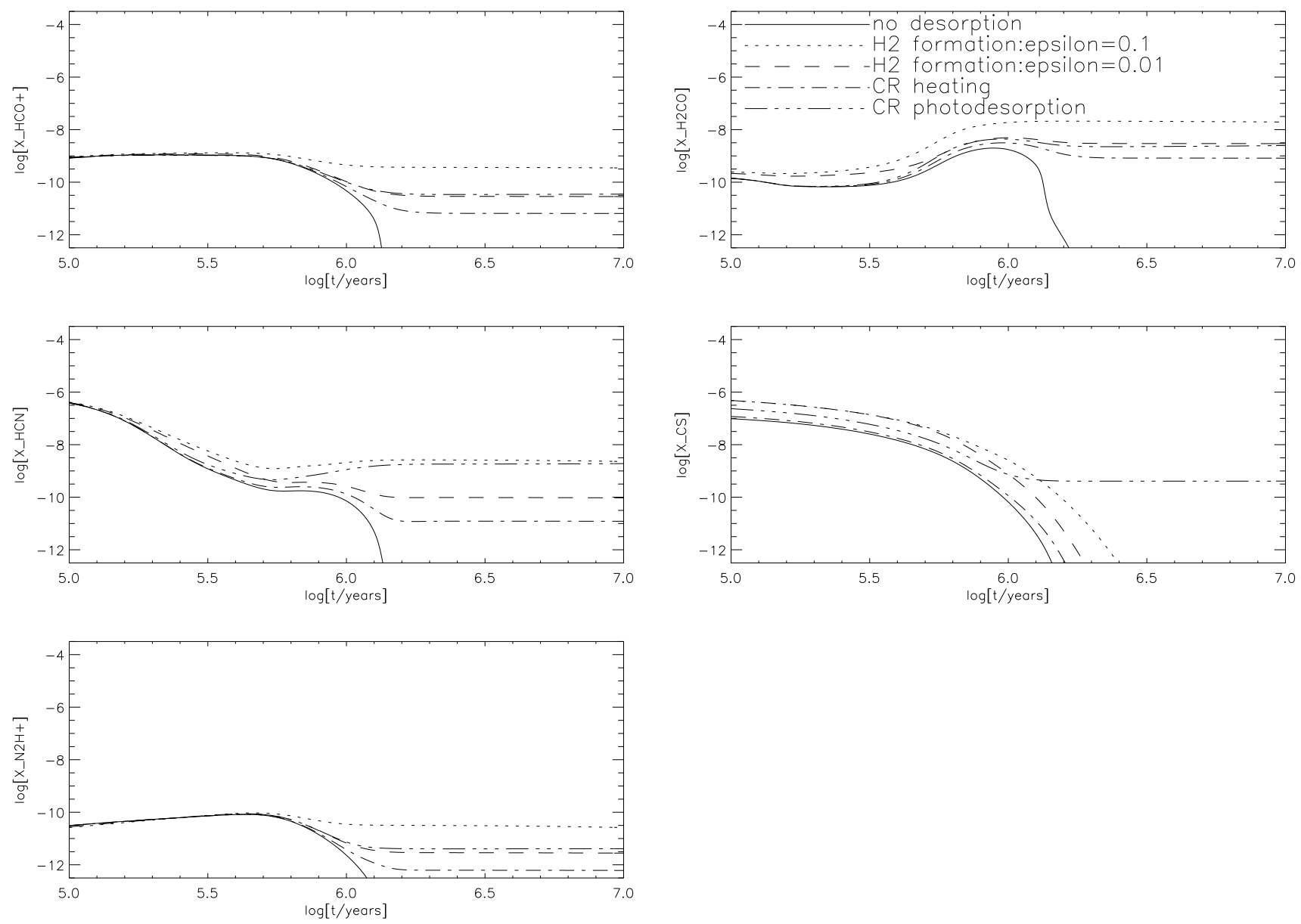

Figure 2. As in Figure 1 but for the molecules $\mathrm{HCO}^{+}, \mathrm{H}_{2} \mathrm{CO}, \mathrm{HCN}, \mathrm{CS}$ and $\mathrm{N}_{2} \mathrm{H}^{+}$.

$B$ due to magnetic fields and other factors (Rawlings et al. 1992). WRW94 used $B=0.7$, a temperature of $10 \mathrm{~K}$, an initial density, $n_{0}$, of $3 \times 10^{3} \mathrm{~cm}^{-3}$ and a final density of $10^{6}$ $\mathrm{cm}^{-3}$. The initial visual extinction of the cloud was $4.4 \mathrm{mag}-$ nitudes. Figure 5 shows the results from our model when it was run with these parameters. We have plotted the abundances of the same molecules that appear in Figure 1 of WRW94, and have converted time to radius as they did to allow for easy comparison between our results and theirs. Even though the chemical reaction network in our model is much more complicated than in the WRW94 model, both models agree that desorption has the greatest effect on $\mathrm{NH}_{3}$ and HCN. Also, under these conditions, we agree that the other mechanisms (direct cosmic ray heating and cosmic ray photodesorption) do not significantly affect the abundances of these molecules.

\subsection{Comparison to WM98}

WM98 carried out an investigation in which they tested the three desorption mechanisms used in our model, as well desorption by the explosive chemical reactions of radicals. Their model used similar physical conditions to ours, except that they adopted a lower density $\left(n_{\mathrm{H}}=2 \times 10^{4} \mathrm{~cm}^{-3}\right)$. They found that models that include desorption by $\mathrm{H}_{2}$ formation (with values of $\varepsilon$ ranging from 0.1 to 1.0 ) gave the best agreement to the observed gas phase abundances in TMC-1.

This actually agrees well with our model, since we also find that desorption by $\mathrm{H}_{2}$ formation with $\varepsilon=0.1$ is the most efficient mechanism. WM98 concluded, however, that desorption by direct cosmic ray heating and cosmic ray photodesorption were too inefficient because they generally under-predicted the gas phase abundances. Thus, for example, CO, was found to be underabundant by approximately two orders of magnitude at an age of 5 Myr using the cosmic ray induced mechanisms. We agree that, when comparing the desorption mechanisms using our standard values of $\varepsilon$, $\phi$ and $Y$, desorption by $\mathrm{H}_{2}$ formation can indeed maintain gas-phase abundances up to two orders of magnitude higher than the other mechanisms. But, since these parameters are so uncertain, we believe it is too early to confidently rule out cosmic ray heating and cosmic ray photodesorption as important desorption mechanisms.

\subsection{Comparison to HW90}

In their study, Hartquist \& Williams (1990) carried out a purely theoretical analysis of cosmic ray induced desorption. They argued that direct cosmic ray heating would not be able to maintain significant gas-phase $\mathrm{CO}$ abundances be- 

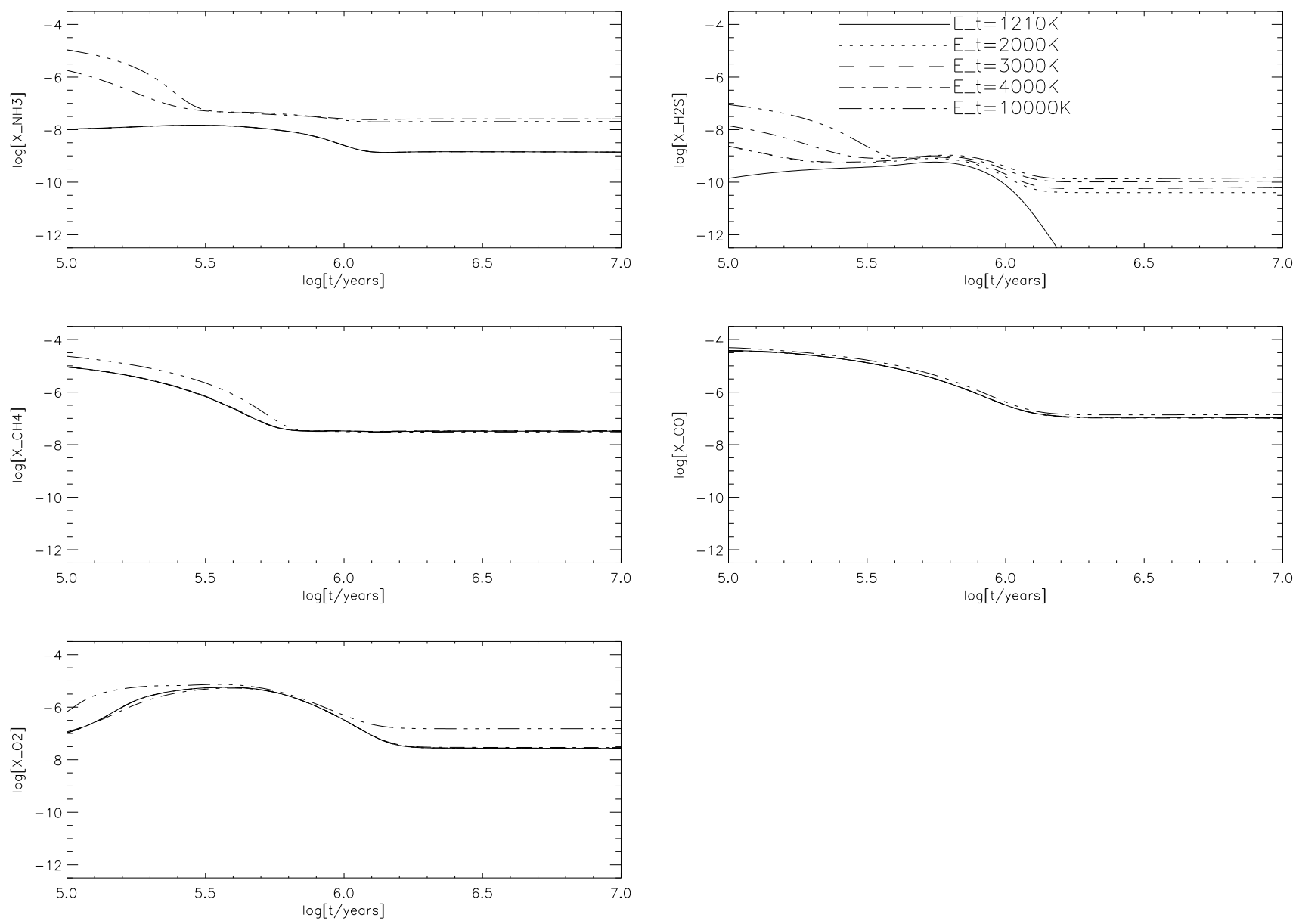

Figure 3. The effect of varying $E_{\mathrm{t}}$ for desorption via $\mathrm{H}_{2}$ formation with $\varepsilon=0.01$, in a static cloud of density $n_{\mathrm{H}}=10^{5} \mathrm{~cm}^{-3}$ at 10 $\mathrm{K}$, for the molecules $\mathrm{NH}_{3}, \mathrm{H}_{2} \mathrm{~S}, \mathrm{CH}_{4}, \mathrm{CO}$ and $\mathrm{O}_{2}$

cause the desorbed $\mathrm{CO}$ would be destroyed by reactions with $\mathrm{He}^{+}$(leading to the formation of hydrocarbons). Their analytical calculation of the gas-phase CO abundance at high densities gives the result

$n_{g}(\mathrm{CO}) \propto e^{-\beta t}$,

where $\beta$ depends on the rates of the reactions

$\mathrm{He}^{+}+\mathrm{CO} \rightarrow \mathrm{C}^{+}+\mathrm{O}+\mathrm{He}$,

$\mathrm{CO}(\mathrm{g}) \rightarrow \mathrm{CO}(\mathrm{s})$

$\mathrm{CO}(\mathrm{s}) \rightarrow \mathrm{CO}(\mathrm{g})$

$\mathrm{He}+\mathrm{CR} \rightarrow \mathrm{He}^{+}+\mathrm{e}+\mathrm{CR}$

and

$\mathrm{He}^{+}+\mathrm{H}_{2} \rightarrow \mathrm{H}^{+}+\mathrm{H}+\mathrm{He}$.

Reactions (8) to (10) are the main formation and destruction routes for $\mathrm{CO}$, with reactions (8) and (10) referring to the freeze-out and desorption of CO. Reactions (11) and (12) are assumed to be the main formation and destruction routes of $\mathrm{He}^{+}$at high densities.

Hartquist \& Williams (1990) calculated $\beta$ to be

$\beta^{-1} \approx 3 \times 10^{6} \mathrm{yr}\left(n_{\mathrm{H}} / 10^{6} \mathrm{~cm}^{-3}\right)$.
However, when we redo the calculation with the (updated) rate coefficients for these reactions used in our model, we obtain values of $\beta^{-1}$ that are a factor almost six orders of magnitude larger. This implies that significant gas-phase CO abundances can be maintained over the lifetime of a typical cloud. The difference arises because we have used a much smaller desorption rate for CO (given by equation (3)). So, in fact, using a smaller desorption rate allows for a more controlled release of $\mathrm{CO}$ into the gas phase and thus enables the $\mathrm{CO}$ to remain in the gas-phase for a longer period of time.

Hartquist \& Williams (1990) also concluded that cosmic ray induced photodesorption would only be effective in regions where the number density is $10^{3} \mathrm{~cm}^{-3}$ or lower. They calculated the ratio at which heavy molecules desorb by cosmic ray induced photodesorption to the rate at which they freeze-out to be

$R \approx\left(\frac{X_{Z}}{10^{-7}}\right)^{-1}\left(\frac{n_{\mathrm{H}}}{10^{5} \mathrm{~cm}^{-3}}\right)^{-1}\left(\frac{\zeta}{10^{-17} \mathrm{~s}^{-1}}\right)$,

where $X_{Z}$ is the fractional abundance of heavy molecules in the gas phase and $\zeta$ is the cosmic ray ionisation rate. This expression implies that cosmic ray photodesorption will be more efficient at low densities. The rates we have used are 

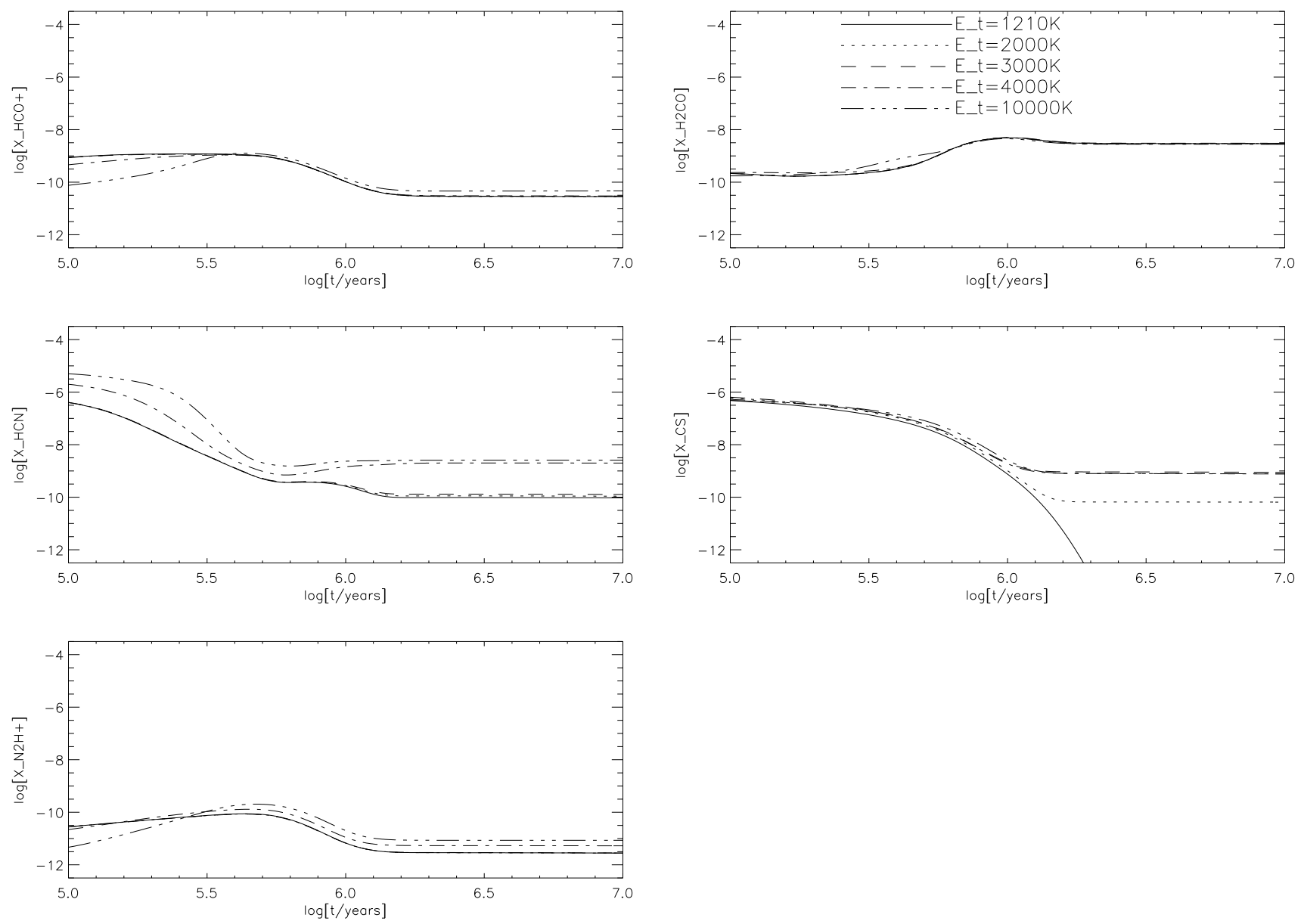

Figure 4. As in figure 3 but for the molecules $\mathrm{HCO}^{+}, \mathrm{H}_{2} \mathrm{CO}, \mathrm{HCN}, \mathrm{CS}$ and $\mathrm{N}_{2} \mathrm{H}^{+}$

consistent with this ratio, but our results clearly indicate that the cosmic ray induced photodesorption mechanism can still maintain significant gas phase abundances of heavy molecules at densities as high as $10^{5} \mathrm{~cm}^{-3}$, even though the efficiency of the process is not at its peak at this density.

\subsection{Discussion of other formulations and assumptions}

As mentioned earlier, other previous models that have included desorption in dark clouds tend to only include desorption by direct cosmic ray heating. One of the most commonly used formulations is that of HH93, who assumed that classical $(\sim 0.1 \mu \mathrm{m})$ grains are impulsively heated by relativistic nuclei with energies $20-70 \mathrm{MeV}$, depositing an average of $0.4 \mathrm{MeV}$ per impact, raising the local (hot spot) temperature of the grain to $70 \mathrm{~K}$. The subsequent (thermal) desorption rate is then calculated from the binding energies of the various molecular species and the cooling profile of the dust grain. In practice, the details of the cooling are simplified to a 'duty cycle' approach, so the desorption rate is proportional to the fraction of time spent by the grains in the vicinity of $70 \mathrm{~K}, f(70 \mathrm{~K}) \sim 3.16 \times 10^{-19}$. The rate coefficient is then given by:

$$
k_{\mathrm{cr}-\mathrm{HH} 93}(i)=f(70 \mathrm{~K}) \nu_{0}(i) \exp \left[-E_{\mathrm{a}}(i) / 70 \mathrm{~K}\right] \quad \mathrm{s}^{-1}
$$

where $\nu_{0}(i)$ and $E_{\mathrm{a}}(i)$ are the characteristic adsorbate vibrational frequency and adsorption energy for species $i$ respectively.

Flower et al. (2005) formulated a similar (but simpler) rate for cosmic ray desorption (in $\mathrm{cm}^{-3} \mathrm{~s}^{-1}$ ) given by:

$R_{\text {crd-FPW05 }}=M_{s}(i)\left\langle\pi a_{\mathrm{g}}^{2} n_{\mathrm{g}}\right\rangle \gamma \exp \left[\frac{-\left(E_{\mathrm{a}}(i)-E_{\mathrm{a}}(\mathrm{CO})\right)}{70 \mathrm{~K}}\right]$

where $\gamma=70 \mathrm{~cm}^{-2} \mathrm{~s}^{-1}$ gives the desorption rate of CO per unit area of dust grains as derived by Léger et al. (1985). This factor takes into account the cosmic ray flux, $F_{\mathrm{cr}}$, and the efficiency parameter, $\phi$, which were explicitly included in our rate. The above rate includes the same exponential factor as in HH93, but does not take into account variations in $\nu_{0}$ for each species.

We decided not to use the above formulations because, bearing in mind the exponential sensitivity of the desorption rate to the ratio of the hot spot temperature to the binding energy, the resulting rates are extremely uncertain and strongly dependent on a number of very poorly constrained free parameters including the grain size and morphology, the molecular binding energies, the rate and energy/mass spectrum of the cosmic rays. 

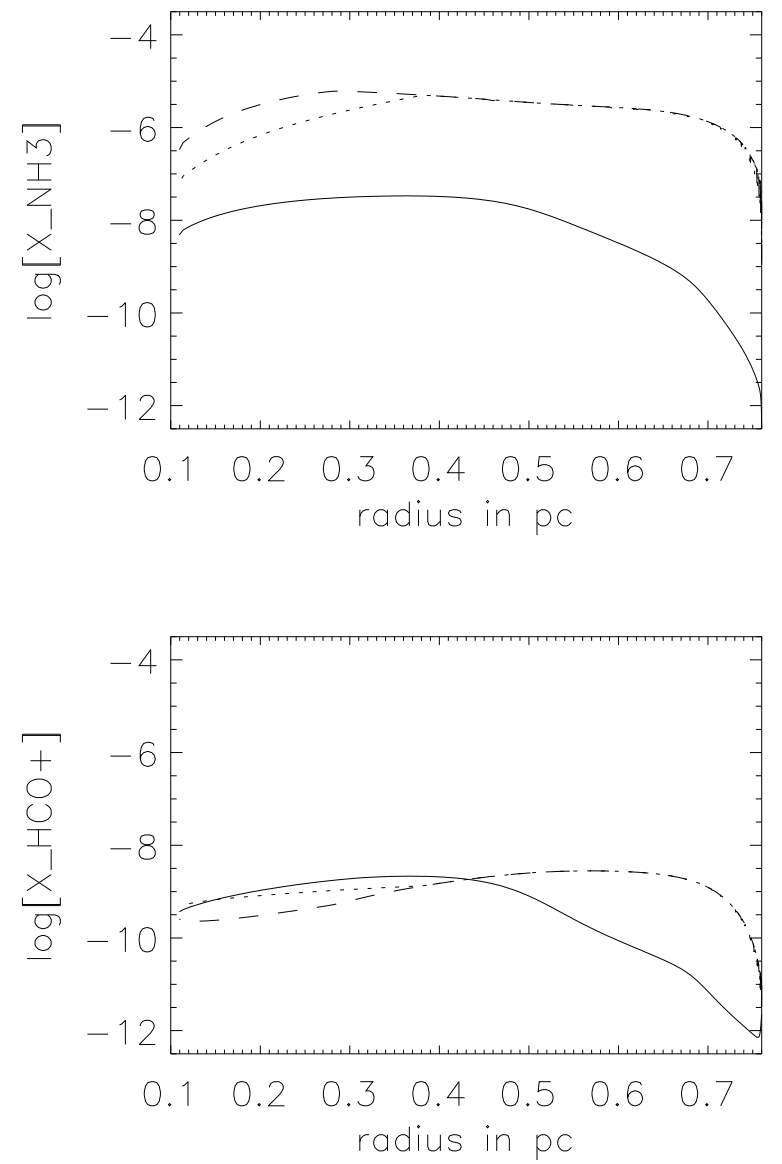
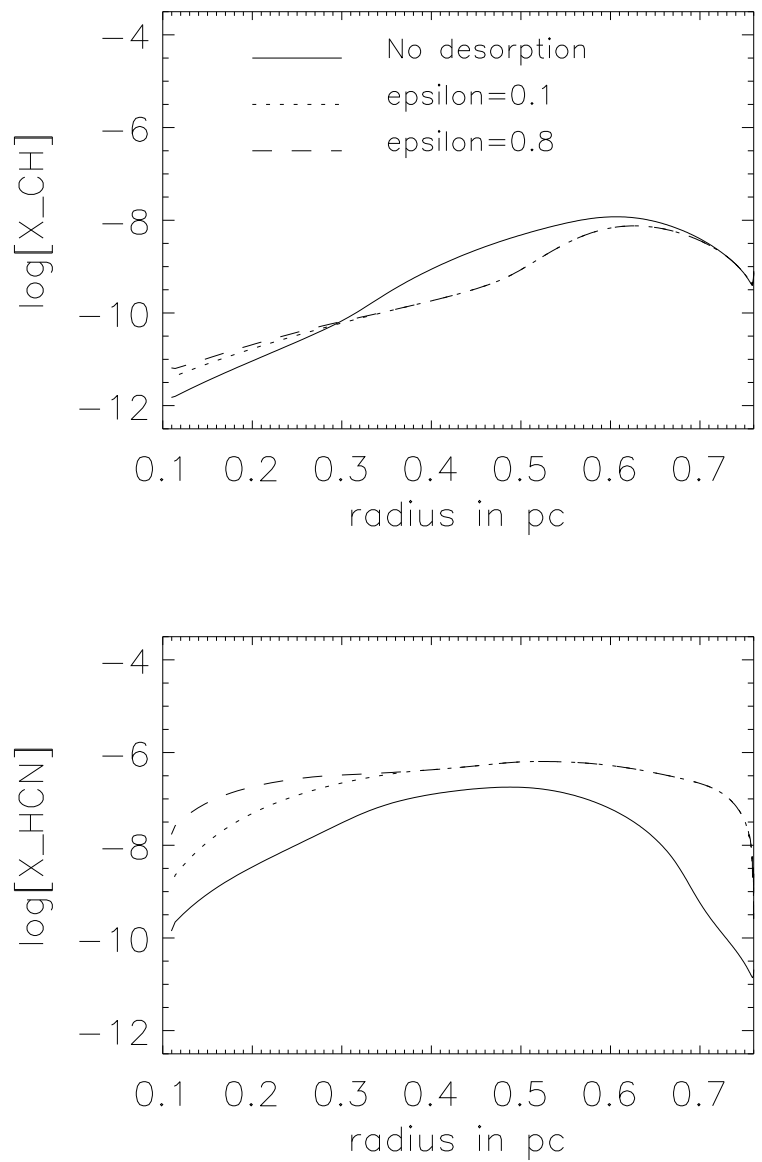

Figure 5. The variation of $\mathrm{NH}_{3}, \mathrm{CH}, \mathrm{HCO}^{+}$and $\mathrm{HCN}$ with radius for a cloud collapsing from $n_{\mathrm{H}}=3 \times 10^{3} \mathrm{~cm}^{-3}$ to $10^{6} \mathrm{~cm}^{-3}$ at $10 \mathrm{~K}$ including non-selective desorption resulting from $\mathrm{H}_{2}$ formation, as in Figure 1 of Willacy et al. (1994b)

We can examine a few of these assumptions in a little more detail:

(i) The formulations given above assume that interstellar dust grains can be thought of as homogeneous, symmetrical (spherical, spheroidal, or cylindrical) entities. In reality, we know that the grains may have very complex morphologies ('fluffy aggregates') with non-uniform thermal properties and composition. We can speculate that these grains therefore consist of poorly thermally connected sub-units, so that both the spot-heating and the whole grain heating rates may be quite different to what is derived for spherical, uniform dust grains.

(ii) Most of these studies also only consider the gas-grain interactions with large $(\sim 0.1 \mu \mathrm{m})$, classical grains. The justification given for this is that the equilibrium temperature of the (cosmic ray heated) smaller grains is too high to allow gas-phase species to freeze-out. However, this differentiation is not apparent in Léger et al. (1985) and there is no direct observational evidence of the universal presence of a population of warm dust grains. The smaller grains present a much larger surface area per unit volume of gas than the classical grains, so this is an important issue.

(iii) Concerning the desorption process itself, the assump- tion is usually made that the mantle ice is pure, so that the adopted binding/desorption energies correspond to the pure substance. Real ices are likely to have complex compositions and temperature-dependent morphologies. Laboratory work Collings et al. 2003, 2004) shows that the desorption characteristics are drastically modified in such circumstances in a way that is very species-dependent. Thus, for example, in a mixed $\mathrm{CO} / \mathrm{H}_{2} \mathrm{O}$ ice, $\mathrm{CO}$ is desorbed in four distinct temperature bands (with $T \sim 25-100 \mathrm{~K}$ ).

(iv) Bringa \& Johnson (2004) performed detailed molecular dynamics calculations of the thermal evaporation process driven by a cylindrical heat pulse, concentrating on a classical grain of $\sim 0.1 \mu \mathrm{m}$, heated by a heavy cosmic ray ion. Even with the assumption of a single desorption energy/threshold for each species they still obtained very different results to $\mathrm{HH} 93$ - most notably the derived $\mathrm{H}_{2} \mathrm{O}$ and $\mathrm{CO}$ desorption rates were found to be an order of magnitude higher and lower, respectively, as compared to HH93. However, even here, the calculations make very general assumptions about the cosmic ray energy spectrum and adopt a spherically symmetric grain with uniform thermal properties.

(v) The issue of the surface chemistry is often not considered in many of the chemical models which use these des- 
orption formulations. This would only be valid if one makes the seemingly unreasonable assumption that the surface residence timescale of adsorbed species is less than the surface migration timescale for reactive species such as hydrogen atoms.

Because of these various uncertainties we must conclude that although approximate quantifications of the rate of desorption driven by cosmic ray grain heating are useful, the numerical details are very poorly constrained and anything more complicated or specific than our simple approach is very hard to justify.

The most important conclusion of this section, therefore, is that due to the considerable uncertainties in the microphysics of ice desorption which are, as yet, very poorly constrained by laboratory and/or theoretical studies, it is very difficult to make qualitative distinctions between different desorption mechanisms: Each of the desorption models discussed above has its own merits, but that there is insufficient information to discriminate or validate these models. However, we also make the point that, through empirically deduced values of the depletions, we can make wellconstrained quantitative estimates of the desorption efficiencies. In the next section, we compare our models to observational results. Thus, by consideration of the deduced desorption efficiencies we are able to comment on the allowed values of the free parameter in each of the desorption models $(\varepsilon, \phi$ or $Y)$ that are consistent with the observations.

\section{COMPARISON WITH OBSERVATIONS}

As a consequence of our poor understanding of the theory of ice desorption, it is proposed that, rather than using desorption efficiencies as an input to the chemical models we invert the process and use whatever observations we can safely interpret to constrain, empirically, the nature and efficiencies of the desorption processes. We can then use that information to predict the chemical behaviours of other species and generate physical diagnostic indicators of the molecular clouds.

Observations of $\mathrm{CO}$ isotopomers in several pre-stellar cores provide strong evidence for the depletion of $\mathrm{CO}$ onto grains. For example, Redman et al. (2002) were able to estimate that at least $90 \%$ of $\mathrm{CO}$ is depleted within $5000 \mathrm{AU}$ of the centre of the pre-stellar core L1689B, by comparing their observations of the $\mathrm{C}^{17} \mathrm{O} J=2 \rightarrow 1$ line with models which included $\mathrm{CO}$ depletion in the centre of the core. Since L1689B is a typical pre-stellar core with a density of $n_{\mathrm{H}}=1.2-1.4 \times 10^{5} \mathrm{~cm}^{-3}$ (Bacmann et al. 2000), it is an ideal candidate with which we can compare our results.

CO depletion has also been estimated in several other pre-stellar cores, such as L1544, L1709A, L310, L328, L429 and Oph D (Bacmann et al. 2002). In these cases, the CO depletion is estimated by comparing the observed ratio, $X$, of the $\mathrm{C}^{17} \mathrm{O}$ and $\mathrm{H}_{2}$ column densities, to the 'canonical' abundance determined by Frerking et al. (1982) towards dark cores, $X_{\text {can }}=4.8 \times 10^{-8}$. The CO depletion factor, $f$, is defined by $X_{\text {can }} / X$, and was found to vary from 4.5 (in L1689B, which appears to be slightly less than the depletion estimated by Redman et al. (2002)) to 15.5 in L429. Since $X_{\text {can }}$ is the value of $X$ obtained in undepleted conditions, and assuming that there is no selective depletion of different isotopomers of $\mathrm{CO}$ (so the fraction of $\mathrm{C}^{17} \mathrm{O}$ depleted onto grains is the same as the fraction of total $\mathrm{CO}$ depleted), then the fraction of $\mathrm{CO}$ depleted onto grains is given by $1-1 / f$. This implies that CO depletion ranges from $78 \%$ to $94 \%$ in these cores. The densities of these cores are all of order $n\left(\mathrm{H}_{2}\right)=10^{5} \mathrm{~cm}^{-3}$ so it is also worth comparing these depletion fractions to our model.

Although our results have indicated that including desorption inhibits full freeze-out allowing abundances to reach an equilibrium, the percentage of $\mathrm{CO}$ frozen-out onto grains is actually greater than $98 \%(f=50)$ for all of the mechanisms investigated, which is consistent with the observations above.

If we assume that L1689B is in a state of equilibrium, the values of $\varepsilon, \phi$ and $Y$ needed to give the $90 \%$ freezeout estimated in L1689B are approximately $0.5,1.3 \times 10^{7}$ and 5.5 respectively. We calculated these values by assuming that freeze-out and desorption of $\mathrm{CO}$ are the main processes governing the $\mathrm{CO}$ abundance, so by equating these rates with $90 \%$ of $\mathrm{CO}$ on the grains and $10 \%$ remaining in the gas phase we can predict the efficiencies needed. We then ran the model with these values of $\varepsilon, \phi$ and $Y$ to confirm that the observed CO depletion is obtained. Since it is possible that L1689B is not in equilibrium and further freeze-out could be achieved, this gives us an upper limit for these parameters.

If we use the values estimated by Bacmann et al. (2002), for L1689B (in which they deduce that CO is depleted by $78 \%$ ) we obtain even higher limits for $\varepsilon, \phi$ and $Y$ of $1.4,3.3 \times 10^{7}$ and 14.0 respectively. For L429, in which a CO depletion of $94 \%$ was deduced, the values for $\varepsilon, \phi$ and $Y$ are reduced to $0.31,7.5 \times 10^{6}$ and 3.2 respectively. The values for all the other cores studied by Bacmann et al. (2002) would lie in between the values for L1689B and L429.

These values are much higher than the estimates used in our model, indicating that if these desorption mechanisms operate then they are very efficient. However, using these observations it is not possible to determine which of the mechanisms is operating, or if it is a combination of all three.

\section{CONCLUSIONS}

There are two major conclusions from this study: Firstly, we have shown that the usual assumption that cosmic ray desorption is the the most effective desorption mechanism in dark molecular clouds is not always valid. All three desorption mechanisms that we have considered (desorption via $\mathrm{H}_{2}$ formation on grain surfaces, direct cosmic ray heating and cosmic ray photodesorption) have been shown to have significant effects on the gas phase abundances in quiescent dark molecular clouds and so should not be neglected in chemical models. These processes all operate on timescales of the order of $\sim 10^{6}$ years. Each of the processes is capable of preventing total freeze-out, but in an equilibrium quiescent dark cloud of density $10^{5} \mathrm{~cm}^{-3}$, the predicted percentage of freeze-out is always greater than $98 \%$. This figure is in good agreement with the observations (e.g. L1689B).

Addressing the specifics of the desorption processes, desorption via $\mathrm{H}_{2}$ formation, if it is efficient $(\varepsilon=0.1)$, is the most effective mechanism. However the complete freeze-out of some species, such as $\mathrm{CS}$ and $\mathrm{H}_{2} \mathrm{~S}$, can only be prevented by the cosmic ray photodesorption mechanism, which is non- 
selective. The relative importance of the three mechanisms appear to be insensitive to variations in the density and the initial atomic to molecular hydrogen ratio. For the selective desorption mechanisms, choosing the threshold adsorption energy, $E_{\mathrm{t}}$, (such that molecules with adsorption energies less than $E_{\mathrm{t}}$ will be desorbed), can have a strong effect on the chemistry, particularly on molecules such as $\mathrm{NH}_{3}, \mathrm{HCN}$, $\mathrm{CS}$ and $\mathrm{H}_{2} \mathrm{~S}$.

Secondly, our understandings of the chemical and physical structure of dust grains and the physical processes which drive desorption are, as yet, very incomplete and a purely theoretical approach to the problem is inadvisable. In this study we have investigated three particular desorption mechanisms (desorption via $\mathrm{H}_{2}$ formation, direct cosmic ray heating and cosmic ray photodesorption) and have used the observed molecular depletions to constrain the poorlydetermined free parameters in desorption processes. This is the first attempt at an empirical determination of the desorption efficiencies. Bearing in mind the huge uncertainties in these efficiencies, this empirical approach is the one that we recommend for use in future studies of interstellar chemistry where gas-grain interactions play an important role.

\section{ACKNOWLEDGEMENTS}

JFR is supported by a PPARC studentship.

\section{REFERENCES}

Aikawa Y., Umebayashi T., Nakano T., Miyama S.M., 1997, ApJ, 486, 51

Allen M., Robinson G.W., 1975, ApJ, 195, 81

Bacmann A., André P., Puget J-L., Abergel A., Bontemps

S., Ward-Thompson D., 2000, A\&A, 361, 555

Bacmann A., Lefloch B., Ceccarelli C., Castets A., Steinacker J., Loinard L., 2002, A\&A, 389, L6

Bringa E.M., Johnson R.E., 2004, ApJ, 603, 159

Cecchi-Pestellini C., Aiello S., 1992, MNRAS, 258, 125

Creighan S.C., Perry J.S.A, Price S.D., 2006, JChemPhys, 124, art. no 114701

Collings, M.P., Dever, J.W., Fraser, H.J., McCoustra, M.R.S., 2003, ApJ., 583, 1058

Collings, M.P., Anderson, M.A., Chen, R., Dever, J.W., Viti, S., Williams, D.A., McCoustra, M.R.S., 2004, Mon. Not. R. Astron. Soc., 354, 1133

Duley W.W., Jones A.P., Whittet D.C.B., Williams D.A., 1989, MNRAS, 241, 697

Duley, W.W., Williams, D.A., 1993, Mon. Not. R. Astron. Soc., 260, 37

Evans N.J., Rawlings J.M.C., Shirley Y.L., Mundy L.G., 2001, ApJ, 557, 193

Flower D.R., Pineau des Forêts G., Walmsley C.M., 2005, A\&A, 436, 933

Fraser H.J., Collings M.P., McCoustra M.R.S., Williams D.A., 2001, MNRAS, 327, 1165

Frerking M.A., Langer W.D., Wilson R.W., 1982, ApJ, 262, 590

Garrod, R.T., Williams, D.A., Rawlings, J.M.C., 2006, ApJ, 638, 827
Garrod R.T., Wakelam V., Herbst E., 2007, A\&A, 467, 1103

Hartquist T.W., Williams D.A., 1990, MNRAS, 247, 343 (HW90)

Hasegawa T.I., Herbst E., 1993, MNRAS, 261, 83 (HH93)

Léger A., Jura M., Omont A., 1985, A\&A, 144, 147

Le Teuff Y.H., Millar T.J., Markwick A.J., 2000, A\&AS, 146, 157

Lee J-E., Evans N.J., Shirley Y.L., Tatematsu K., 2003, ApJ, 583, 789

Morfill G.E., Volk H.J., Lee M.A., 1976, J. Geophys. Res., 81,5841

Prasad S.S., Tarafdar S.P., 1983, ApJ, 267, 603

Rawlings J.M.C., Hartquist T.W., Menten K.M., Williams D.A., 1992, MNRAS, 255, 471

Redman M.P., Rawlings J.M.C., Nutter D.J., WardThompson D., Williams D.A., 2002, MNRAS, 337, L17

Roberts H., Herbst E., Millar T.J., 2004, A\&A, 424, 905

Ruffle D.P., Herbst E., 2000, MNRAS, 319, 837

Shalabiea O.M., Greenberg J.M., 1994, A\&A, 290, 266

Shen C.J., Greenberg J.M., Schutte W.A., van Dishoeck E.F., 2004, A\&A, 415, 203

Smith I.W.M., Herbst E., Chang Q., 2004, MNRAS, 350, 323

Sofia U.J., Meyer D.M., 2001, ApJ, 554, L221

Viti S., Collings M.P., Dever J.W., McCoustra M.R.S., Williams D.A., 2004, MNRAS, 354, 1141

Wakelam V., Herbst E., Selsis F., 2006, A\&A, 451, 551

Willacy K., Williams D.A., Duley W.W., 1994a, MNRAS, 267, 949

Willacy K., Rawlings J.M.C., Williams D.A., 1994b, MNRAS, 269, 921 (WRW94)

Willacy K., Millar T.J., 1998, MNRAS, 298, 562 (WM98)

This paper has been typeset from a $\mathrm{TEX}_{\mathrm{E}} \mathrm{X}$ L $\mathrm{T}_{\mathrm{E}} \mathrm{X}$ file prepared by the author. 